Received Date : 30-Oct-2015

Revised Date : 13-Jan-2016

Accepted Date : 18-Mar-2016

Article type : Regular Article

\title{
Preterm cranial ultrasound scanning is both feasible and effective in a middle- income country
}

\author{
Pavel A Mazmanyan ${ }^{1,2}$, Knarik V Nikoghosyan ${ }^{2}$, Varsenik V Kerobyan ${ }^{1,2}$, \\ Kathy $\mathrm{J} \mathrm{Mellor}^{3}$, Jesus Diez-Sebastian ${ }^{4}$, Miriam Martinez-Biarge ${ }^{5}$, \\ Frances M Cowan ${ }^{5}$
}

1 Research Centre of Maternal and Child Health Protection, Yerevian, Armenia 2 Department of Neonatology, Yerevan State Medical University, Armenia 3 BirthLink, UK

4 Biostatistics Department, La Paz University Hospital, Madrid, Spain

5 Department of Paediatrics, Imperial College London, UK

Correspondence to:

Dr Pavel Mazmanyan

Research Centre of Maternal and Child Health Protection

Mashtots Avenue. 22, 0002

Yerevan, Armenia

Email: pavelart@gmail.com

Keywords: cranial ultrasound imaging, intraventricular haemorrhage, middleincome country, neonatal intensive care, preterm.

This article has been accepted for publication and undergone full peer review but has not been through the copyediting, typesetting, pagination and proofreading process, which may lead to differences between this version and the Version of Record. Please cite this article as doi: 10.1111/apa.13411

This article is protected by copyright. All rights reserved. 
Running title: Preterm cranial ultrasound in Armenia

\section{ABSTRACT}

Aim: Cranial ultrasound is seldom used in middle-income countries and the burden of preterm brain injury and its relationship to perinatal data is unknown. We assessed cranial ultrasound abnormalities in very low birth weight (VLBW) infants and correlated the findings with perinatal data.

Methods: VLBW Armenian infants receiving neonatal intensive care in 2012 were scanned from birth to term-equivalent age (TEA). Clinical data were collected prospectively.

Results: We studied 100 VLBW infants with a median gestation of 30 weeks. Periventricular white matter echogenicity (PVE) lasting more than two weeks was seen in 34 infants, grade-III intraventricular haemorrhage (IVH) in 10, haemorrhagic parenchymal infarction (HPI) in seven and cystic periventricular leukomalacia in two. Caudo-thalamic notch echogenicity appeared in 36 infants after two to three weeks, with cystic transformation in 22. At TEA, 17 infants had persisting PVEs and 55 had increased basal ganglia/thalamic (BGT) echogenicity. Lack of antenatal steroids was significantly associated with IVH and HPI and intubation at birth with IVH. Late BGT echogenicity was generally seen in infants without perinatal problems.

Conclusion: Our study demonstrated that cranial ultrasound can be used effectively in a middle-income country to identify high-risk infants and monitor quality of care.

\section{Key notes}

- Cranial ultrasound is seldom used in middle-income countries and the burden of preterm brain injury and its relation to perinatal data is unknown.

- Our study of very low birth weight Armenian infants showed that abnormalities were more common in infants who did not receive antenatal steroids and those 
who required resuscitation.

- Routine cranial ultrasound imaging in middle-income countries should be used to identify high-risk infants and monitor quality of care.

\section{INTRODUCTION}

An increasing number of preterm infants are receiving neonatal intensive care in middle-income and emerging countries (1). This has resulted in a decreased neonatal mortality rate among these vulnerable babies $(2,3)$, but concerns over high neurodevelopmental impairment rates in postneonatal survivors are rising (1). Preterm birth has been associated with increased worldwide risks of cerebral palsy, intellectual disabilities and psychiatric disorders $(1,4)$.

Identifying preterm infants at high risk of neurodevelopmental impairment is critical in order to plan appropriate follow up and rehabilitation programmes and to reduce the burden of childhood disability. Cranial ultrasound allows early bedside detection of most major intracranial lesions, which are known to be the best predictor of cerebral palsy in preterm infants (5). Although few studies have reported the incidence of major intracranial lesions in cohorts of preterm infants in low and middle-income countries (6-8), cranial ultrasound is seldom used in these settings. No previous studies have assessed the feasibility and clinical usefulness of a routine cranial ultrasound protocol for the detection and follow up of preterm brain injury in low and middle-income countries.

A significant decline in neonatal mortality rates was observed in many middleincome countries between 1990 and 2013, including 42\% in South Asia and 47\% in the Middle East and North African regions (3). In Armenia, neonatal mortality fell by 47\% from 17 to eight per 1,000 live births between the late 1990 s and 2005 (9). Although neonatal intensive care was introduced in Armenia in 1987, bedside cranial ultrasound only became available in 2012.

This article is protected by copyright. All rights reserved. 
The aim of our study was to evaluate the feasibility of a sequentially-acquired neonatal cranial ultrasound scan programme in a neonatal intensive care unit (NICU) in a middle-income country. We also aimed to assess the cranial ultrasound findings of a cohort of very low birth weight (VLBW) Armenian infants and to correlate these findings to their perinatal data.

\section{PATIENTS AND METHODS}

The study group included preterm infants born at $\leq 32$ completed weeks of gestation or weighing $\leq 1,500 \mathrm{~g}$ who were admitted between February and December 2012 to the NICUs of the Research Centre of Maternal and Child Health Protection and the Institute of Reproductive Health, Perinatology, Obstetrics and Gynecology, Yerevan, Armenia. Both are tertiary referral centers with approximately 3,500 deliveries and 300-400 NICU admissions a year. The NICUs provide conventional respiratory support and continuous positive airway pressure and, in general, surfactant is available. Parenteral nutrition is only provided in selected cases.

Infants were studied prospectively using serial cranial ultrasound. Perinatal and clinical data were collected prospectively. Gestational age was determined from first trimester ultrasounds and all infants also had a Ballard examination. Ethical approval was obtained from the ethics committees of both hospitals. Both oral and written parental consent was obtained.

\section{Ultrasound examinations}

Our aim was to perform cranial ultrasound examinations at four time points: as soon as possible after admission, two to three weeks after birth, before discharge home or to another unit - which was usually at 34-37 weeks of post-menstrual age - and at term-equivalent age (TEA). Additional scans were performed if the infant's clinical condition deteriorated or there were specific neurological concerns.

This article is protected by copyright. All rights reserved. 
All the infants were scanned by a single investigator (PM) using a hand-portable z.one ultra Convertible Ultrasound ${ }^{\mathrm{TM}}$ System (Zonare Medical Systems Inc, California, USA) with a 7.5 and $8 \mathrm{MHz}$ frequency microconvex C9-4t tightly curved array transducer. The images were stored digitally and assessed using OsiriX, a free open-source programme available for Apple Mac computers. The protocol included a minimum of five coronal views, one mid-sagittal, and two left and two right parasagittal views, according to Meijler et al (10). The scans were assessed for normal anatomy, ventricular size, germinal matrix-intraventricular haemorrhage (GMH-IVH), intraparenchymal lesions, white matter echogenicity including periventricular echodensities (PVE), cystic periventricular leukomalacia, subependymal echogencity and cysts independent of $\mathrm{GMH}$, lenticulostriate vasculopathy, cerebellar and developmental anomalies. GMH-IVH was classified according to Volpe (11) and PVE and cystic periventricular leukomalacia according to de Vries et al (12). All images were first assessed by one of the authors (PM) and then reviewed by another author (FC) who has many years experience in cranial ultrasound imaging and interpretation. Both assessors were blinded to the outcome data and a consensus on the diagnoses was reached.

The cranial ultrasound data was analysed sequentially within each infant and together within each acquisition period. The imaging data were also compared to the demographic and clinical data listed in Table 1, taking into account the time of the scan and the clinical event. Data were analysed using IBM SPSS Statistics 20 for Mac and SPSS v.11.5 (SPSS Inc, Illinois, USA). The associations between perinatal and clinical factors and cranial ultrasound findings were assessed using Fisher's exact two-sided test for categorical variables and the Mann-Whitney test for quantitative variables. Differences with a $p$ level of $<0.05$ were considered significant. Multivariable regression analysis was performed to determine 
independent variables associated with neonatal death. Variables associated with a univariable $p$ value of $<0.10$ were included in the multivariable analysis.

\section{RESULTS}

\section{Patients}

During the study period 132 VLBW infants born at $\leq 32$ weeks of gestation and, or, weighing $\leq 1,500 \mathrm{~g}$ were admitted to the NICUs of the two units and the parents of 100 infants gave permission for them to be included in the study. All were inborn infants. There were no significant differences in terms of gestational age, birth weight, sex, maternal age, type of delivery and Apgar scores between the 100 infants who were enrolled and the 32 who were not enrolled in the study (data not shown). Of the 32 neonates who were not enrolled, 16 died before scanning and 16 were born when one of the authors (PM) was away. Infants who were not enrolled had a higher mortality rate $(50 \%)$ than the infants who were enrolled (19\%) $(p=0.002)$. The main demographic, perinatal and clinical characteristics of the enrolled infants are shown in Table 1.

\section{Cranial ultrasound findings}

A total of 341 scans were obtained and the median number of scans for each infant surviving to TEA ( $n=81$ ) was four (range 1-7) and in the 19 infants who died it was two (range 1-4). We obtained 99 scans in the first 10 days (median day four, range 1-10), 68 between days 11-21 (median day 17, range 11-21), 100 at discharge (median day 37, range 22-68) and 74 at TEA (median day 87, range 33-191).

\section{Early scans at 1-10 days}

There were 99 early scans carried out at 1-10 days. Mild unilateral or bilateral lateral ventriculomegaly (VM), without GMH-IVH, was seen in 14 infants on their first scan: in five the VM persisted to TEA and in one it was associated with congenital 
cytomegalovirus infection. The vermis was small inferiorly in one infant and oddly shaped in another. GMH-IVH was seen in 17 infants - 10 had grade III (IVH III), three had grade II (IVH II) and four had grade I (IVH I, referred to as GMH) - and in all but one infant this occurred in the first 10 days after birth. In two infants it was thought to have occurred just before delivery. Haemorrhagic parenchymal infarction (HPI) was seen in seven infants, six of whom also had grade III GMH-IVH. Thus, the combined incidence of grades II and III IVH and HPI was 11\%. Bilateral PVEs were seen in $34 \%$ of infants. Two infants had sub-ependymal cysts, two had lenticulostriate vasculopathy and three had choroid plexus cysts. These findings are summarised in Table 2 and illustrated in Figure 1.

\section{Later white matter and haemorrhagic findings}

We looked at white matter and haemorrhagic findings on the second and third scans, with evolution to term age. One infant was found to have a GMH-IVH in this period that was not seen on the first scan. Post-haemorrhagic ventriculomegaly developed in three infants, none of whom required a shunt. Of the seven infants with an HPI, five died and the other two developed a porencephalic cyst at the site of the lesion. Atypical focal parenchymal haemorrhage was found in three infants (Figure 2). Cerebellar haemorrhage was detected in five infants, all of whom had grade III GMH-IVH and three had an HPI and one had an atypical haemorrhage. Two of these infants died. Bilateral white matter PVEs, seen on the first scan, persisted for four to 10 weeks in 20 infants and until TEA in 17. The PVEs were patchy, suggestive of punctate white matter lesions, in 11 . Only two infants developed cystic periventricular leukomalacia, first seen on day 18 in one and on day 42 in the other.

This article is protected by copyright. All rights reserved. 


\section{Findings at term-equivalent age}

TEA scans were available for 68 infants. The main finding was mild ventriculomegaly in 19 infants $(15,79 \%)$, which was mostly not associated with $\mathrm{GMH}-\mathrm{IVH}$, and had been present on the initial scan in one-third of cases. Other findings were widening of the extracerebral space and interhemispheric fissure, thinning of the corpus callosum and the appearance of a reduced white matter volume not seen on earlier scans. An unexpected finding was diffuse echogenicity in the BGT in over half the infants. In some it was already evident by 36 weeks of postmenstrual age (Figure 2) and in about a third of cases it was associated with an increase in extracerebral space and widening of the interhemispheric fissure. Scans were considered normal in 11 infants (Table 3).

\section{Abnormal echogenicity and cysts in the caudothalamic notch}

Sub-ependymal cysts adjacent to the anterior horns of the lateral ventricles were seen as isolated findings in three infants. Small choroid plexus cysts were seen in six infants, of whom five also had late BGT echogenicity and four developed widened extracerbral spaces $(p<0.05)$. No other associations were found. Lenticulostriate vasculopathy was seen in seven infants, two of whom had later evolving caudothalamic notch echoes/cysts, three had PVEs lasting more than four weeks, five had late BGT echogenicity, three had ventriculomegaly at term, two had widened extracerbral spaces and one had a thin corpus callosum.

Echogenicity in the caudothalamic notch, not typical of $\mathrm{GMH}$, was seen to evolve in 36 infants, usually after the second week. It was more pronounced on a later scan and cysts developed in $22(55 \%)$ cases (Figure 2). Ten of these infants were tested for cytomegalovirus from Guthrie blood spots from the first postnatal week and the test was negative in nine cases. The one infant who tested positive had early scan findings that were highly suggestive of congenital cytomegalovirus infection with caudothalamic notch cysts, strands across the ventricle and PVEs. 


\section{Ultrasound finding in suspected perinatal asphyxia}

Perinatal asphyxia was initially suspected in 15 infants, defined as no early spontaneous respiration, being unresponsive or excessively floppy and, or, having seizures, although clearly it was unlikely that this was indeed the diagnosis. Of the 15, eight were born by emergency Caesarean section and the Apgar scores were $\leq 3$ at one minute in 13 infants and $<3$ at five minutes in six infants. The cord $\mathrm{pH}$ values were not available. None of these infants had signs of neonatal encephalopathy and none developed the BGT echogenicity that is typical of acute perinatal asphyxia (13). However seven died (47\%), all with infection related problems. Two developed severe IVH/HPI, two had PVEs, one had amorphous appearing white matter and two had fairly normal scans. Of the remaining eight infants, five developed ill-defined hazy looking white matter with PVEs, one of whom developed cystic periventricular leukomalacia, another had a GMH and a focal BGT lesion, one had a small focal trigonal lesion, two had large IVH/HPI and one only had an echogenic looking brainstem.

\section{Ultrasound findings in infants who died}

Of the 19 infants who died, seven were initially described as having asphyxia but with clinical diagnoses of infection. The other 12 did not have severe cranial ultrasound findings. The most frequent cause of death was infection $(n=14,74 \%)$ : six infants had necrotising enterocolitis, eight had sepsis and seven of these infants also had an HPI and, or, IVH III. Several perinatal and clinical factors were associated with death on the univariable aanalysis, but on multivariable analysis only lower gestation age $(p=0.008)$, lower birth weight $(p=0.01)$ and lack of antenatal steroids $(p=0.06)$ remained significant. HPI was the only imaging factor significantly associated with death $(\mathrm{p}=0.002)$.

This article is protected by copyright. All rights reserved. 


\section{Perinatal factors associated with cranial ultrasound findings}

Risk factors related to early cranial ultrasound findings were non administration of antenatal steroids, which was significantly associated with IVH II and III (84\% versus $53 \%, p=0.037)$, HPI (100\% versus 54\%, $\mathrm{p}=0.019)$ and intubation at birth, which was associated with IVH II and III (15.5\% versus $1 \%, \mathrm{p}=0.044)$.

The occurrence of late caudothalamic notch echoes and related cyst formation was significantly associated with the number of days on oxygen $(p=0.003)$, number of days on continuous positive airways pressure $(p=0.043)$, greater prematurity $(p=0.018)$ and more blood transfusions $(p=0.033)$. A later widened extracerebral space and ventriculomegaly at TEA, not associated with GMH-IVH, were associated with prolonged rupture of the membranes $(p=0.005)$ and suspected sepsis $(p=0.029)$, respectively, and ventriculomegaly, only seen at TEA, was associated with necrotising enterocolitis $(p=0.039)$.

Late-occurring BGT echoes were inversely associated with most other abnormal cranial ultrasound findings and these findings were significant with IVH III $\quad(p=0.005)$ and HPI $(p=0.02)$. These infants were older, with a gestational age of 31 versus 28.9 weeks $(p<0.001)$ and had higher one-minute Apgar scores (mean 4.6 versus 3.97 $\mathrm{P}=0.024)$ compared to infants without these findings.

\section{DISCUSSION}

Our study shows that it is feasible to obtain high-quality cranial ultrasound images in preterm infants admitted to a NICU in a middle-income country. Although no decision to discontinue intensive care was made based on cranial ultrasound findings, they provided information for prognosis, counselling and planning further care. After this study finished, routine cranial ultrasound became standard practice in our units. In addition we are now conducting neurodevelopmental assessments at 
two-years of age in this cohort as the first step in developing a follow-up programme for preterm infants in Armenia.

We found cranial ultrasound abnormalities in $93 \%$ of VLBW infants. GMH-IVH grades II and III occurred in $13 \%$, with just over $50 \%$ of these infants developing an $\mathrm{HPI}$. Although these figures were not dissimilar to rates reported in western countries (14-16), the infants in those studies were of significantly lower gestation and thus it is likely that the real incidence of GMH-IVH and HPI, after adjusting for gestational age, was higher in our cohort.

Bilateral PVEs were common, lasting more than two weeks in $34 \%$ of infants, four10 weeks in $27 \%$ and up to TEA in $17 \%$. Although this rate was higher than reported in western countries $(5,14-16)$, the incidence of $\mathrm{CPVL}$ was low and identical to rates reported elsewhere $(5,14-21)$.

An interesting finding was the development of echogenicity in the caudothalamic notch in 36 infants and the later development of cysts in 22 of these 36 . Leijser et al found germinolytic cysts in $5.3 \%$ of admission scans in 113 VLBW infants in the Netherlands and in $4.4 \%$ of scans at TEA (15). We excluded congenital cytomegalovirus infection in nine of the 10 infants tested. In general, caudothalamic notch echogencity started to occur in the second to third post-natal week, rather early to be a sign of postnatal cytomegalovirus acquisition, which was not tested for. Use of maternal breast milk in the first postnatal week was usual and hence a possible source of cytomegalovirus infection.

The unusual observation of late diffuse BGT echogenicity, was inversely associated with most abnormal cranial ultrasound findings and this inverse association was statistically significant in the case of IVH III and HPI. van Wezel-Meijler et al 
reported diffuse, homogeneous bilateral BGT echogenicity in $92 \%$ of 130 VLBW infants, but those echogenicities were always seen early, they diminished with age and TEA MRI did not show any BGT abnormalities. The authors concluded that this BGT echogenicity was a prematurity-related normal maturational phenomenon (22). Another pattern of BGT echogenicity, which was not neccesarily bilateral but frequently associated with other brain lesions in more immature and unstable infants, was also described in $10 \%$ of VLBW infants, (23-24). The pattern of BGT echogenicity in our cohort seems different to both of these studies, but the high incidence, the association with better conditions at birth and the inverse correlation with other imaging abnormalities suggest that they may have been a transient nonpathological finding. Unfortunately we could not assess the BGT using MRI and neurodevelopmental outcome information is not yet available.

We found an association between the lack of administration of antenatal steroids and neonatal death and the presence of GMH-IVH grades II-III and HPI and also between intubation at birth and the occurrence of IVH. In a randomised trial of an intervention designed to increase the use of antenatal steroids in low and middleincome countries, the intervention was associated with a significant increase in neonatal mortality and in maternal infection rates (25). However, the majority of the infants in the trial did not have access to intensive care. Our findings are in keeping with other studies performed in similar settings to ours (26-27). They suggest that, in the context of adequate prenatal and neonatal intensive care, antenatal steroids and the use of non-invasive techniques at resuscitation, such as facemask ventilation and continuous positive airway pressure, are beneficial for VLBW infants in middleincome countries $(27,28)$.

This article is protected by copyright. All rights reserved. 
Perinatal asphyxia was initially diagnosed in 15 infants because they were born in a poor condition, but the reason for their depression at birth was infection in most cases. In settings where cord $\mathrm{pH}$ and brain monitoring are not available, it is not uncommon for these infants to be mis-classified.

This study had some limitations. As there was only one operator and one ultrasound machine for the two units, not all of the infants had four scans and only $19 \%$ of the infants had a scan on admission, although $52 \%$ had had a scan by day three. Compared to other preterm cohorts, our infants were of older gestational age, making comparisons between cranial ultrasound abnormality rates difficult. The strengths of the study were the large number of scans performed in a homogenous group of VLBW infants receiving intensive care in a middle-income setting. The scan quality was very high, performed and assessed by one experienced neonatologist and independently reviewed by a perinatal neurologist with many years of experience in cranial ultrasound imaging. Clinical data were prospectively collected and were complete in most cases.

\section{CONCLUSION}

Our study has shown that it is possible to obtain high-quality cranial ultrasound images in a middle-income setting that can be used for diagnosis and as one means of monitoring the quality of care. Our results show a higher incidence of non-cystic white matter injury, caudothalamic notch echogenicity and cysts and likely GMH-IVH than reported in high-income countries. These findings are in agreement with studies describing higher rates of neurodevelopmental impairments among preterm infants in middle and low-income countries $(1,29)$ and stress the need to identify infants at risk during the neonatal period and to continuously monitor the quality of care in these settings (1). Cranial ultrasound imaging could be a very useful tool for this purpose, as it is safe and relatively inexpensive, can be performed at the 
bedside as often as necessary and allows the detection of most major brain lesions (10). Performing reliable cranial ultrasound scans requires training, but no more than the training required in other areas of neonatal intensive care. The impact of early and accurate detection of significant brain abnormalities and normality on the health and long-term neurodevelopmental status of preterm infants should not be underestimated.

\section{Acknowledgements}

The authors are grateful to Dr Jean Luc Murk, University Medical Centre, Utrecht, The Netherlands, for testing for cytomegalovirus, to the nursing staff of both neonatal intensive care units and to Dr Serine Meliksetyan, head of the neonatal intensive care unit at the Institute of Reproductive Health Perinatology, Obstetrics and Gynecology, Yerevan, for support during this study.

\section{Funding}

BirthLink, UK and Vivacell-MTS Armenia funded the cranial ultrasound scanner and other neonatal equipment.

\section{Conflicts of interest}

The authors have no conflict of interests to declare.

\section{List of abbreviations}

BGT: basal ganglia/thalamic

GMH-IVH: germinal matrix-intraventricular haemorrhage

HPI: haemorrhagic parenchymal infarction

IVH: intraventricular haemorrhage

MRI: magnetic resonance imaging

NICU: neonatal intensive care unit

This article is protected by copyright. All rights reserved. 
PVE: periventricular echogenicity

TEA: term-equivalent age

\section{REFERENCES}

1. Blencowe H, Lee AC, Cousens S, Bahalim A, Narwal R, Zhong N, et al. Preterm birth-associated neurodevelopmental impairment estimates at regional and global levels for 2010. Pediatr Res 2013;74 Suppl 1:17-34.

2. Blencowe H, Cousens S, Oestergaard MZ, Chou D, Moller AB, Narwal R, et al. National, regional, and worldwide estimates of preterm birth rates in the year 2010 with time trends since 1990 for selected countries: a systematic analysis and implications. Lancet 2012;379:2162-72.

3. UNICEF. UNICEF analysis based on United Nations Inter-agency Group for Child Mortality Estimation (UN IGME), as published in UNICEF: Committing to Child Survival: A promise renewed-Progress report 2014, UNICEF, New York 2014.http://data.unicef.org/child-mortality/neonatal\#sthash.H33l7t9d.dpuf (date accessed: May 2015).

4. Marlow N, Wolke D, Bracewell MA, Samara M. Neurologic and developmental disability at six years of age after extremely preterm birth. $N$ Engl $J$ Med 2005;352:9-19.5.

5. De Vries LS, Van Haastert IL, Rademaker KJ, Koopman C, Groenendaal F. Ultrasound abnormalities preceding cerebral palsy in high-risk preterm infants. $J$ Pediatr 2004;144:815-20.

6. Ajayi O, Nzeh DA. Intraventricular haemorrhage and periventricular leukomalacia in Nigerian infants of very low birth weight. West Afr J Med 2003;22:164-6.

7. Kadri H, Mawla AA, Kazah J. The incidence, timing, and predisposing factors of germinal matrix and intraventricular hemorrhage $(\mathrm{GMH} / \mathrm{IVH})$ in preterm neonates. Childs Nerv Syst 2006;22:1086-90.

This article is protected by copyright. All rights reserved. 
8. Badiee Z. Intraventricular hemorrhage in very low birth weight infants. Associated risk factors in Isfahan, Iran. Saudi Med J 2007;28:1362-6.

9. National Statistical Service (NSS) [Armenia], Ministry of Health (MOH), and ORC Macro. 2006. Armenia Demographic and Health Survey 2010. Calverton, Maryland: National Statistical Service, Ministry of Health, and ORC Macro.

10. van Wezel-Meijler G, Steggerda SJ, Leijser LM. Cranial ultrasonography in neonates: role and limitations. Semin Perinatol 2010;34:28-38.

11. Volpe JJ. Neurology of the newborn. 5th ed. Philadelphia: WB Saunders; 2008, page 541.

12. de Vries LS, Eken P, Dubowitz LMS. The spectrum of leukomalacia using cranial ultrasound. Behav Brain Res 1992;49:1-6.

13. Logitharajah P, Rutherford MA, Cowan FM. Hypoxic-ischemic encephalopathy in preterm infants: antecedent factors, brain imaging, and outcome. Pediatr Res 2009;66:222-9. doi: 10.1203/PDR.0b013e3181a9ef34.

14. Miller SP, Cozzio CC, Goldstein RB, Ferriero DM, Partridge JC, Vigneron DB, et al. Comparing the diagnosis of white matter injury in premature newborns with serial MR imaging and transfontanel ultrasonography findings. AJNR Am J Neuroradiol 2003;24:1661-9.

15. Leijser LM, de Bruïne FT, Steggerda SJ, van der Grond J, Walther FJ, van Wezel-Meijler G. Brain imaging findings in very preterm infants throughout the neonatal period: part I. Incidences and evolution of lesions, comparison between ultrasound and MRI. Early Hum Dev 2009;85:101-9.

16. Plaisier A, Raets MM, Ecury-Goossen GM, Govaert P, Feijen-Roon M, Reiss IK et al. Serial cranial ultrasonography or early MRI for detecting preterm brain injury? Arch Dis Child Fetal Neonatal Ed 2015;100:F293-300.

17. Inder TE, Anderson NJ, Spencer C, Wells S, Volpe JJ. White matter injury in the premature infant: a comparison between serial cranial sonographic and MR 
findings at term. AJNR Am J Neuroradiol 2003;24:805-9.

18. Ciambra G, Arachi S, Protano C, Cellitti R, Caoci S, Di Biasi C et al. Accuracy of transcranial ultrasound in the detection of mild white matter lesions in newborns. Neuroradiol J 2013;26:284-9.

19. Bajwa NM, Berner M, Worley S, Pfister RE; Swiss Neonatal Network. Population based age stratified morbidities of premature infants in Switzerland. Swiss Med Wkly 2011 Jun 24;141:w13212..

20. van Haastert IC, Groenendaal F, Uiterwaal CS, Termote JU, van der HeideJalving $\mathrm{M}$, et al. Decreasing incidence and severity of cerebral palsy in prematurely born children. J Pediatr 2011;159:86-91.

21. Horbar JD, Carpenter JH, Badger GJ, Kenny MJ, Soll RF, Morrow KA, et al. Mortality and neonatal morbidity among infants 501 to 1500 grams from 2000 to 2009. Pediatrics 2012;129:1019-26.

22. Van Wezel-Meijler G, Leijser LM, Wiggers-de Bruïne FT, Steggerda SJ, van der Grond J, Walther FJ. Diffuse hyperechogenicity of basal ganglia and thalami in preterm neonates: A physiologic finding? Radiology 2011;258:944-950.

23. Leijser LM, Klein RH, Veen S, Liauw L, Van Wezel-Meijler G. Hyperechogenicity of the thalamus and basal ganglia in very preterm infants: radiological findings and short-term neurological outcome. Neuropediatrics 2004;35:283-9.

24. Soghier LM, Vega M, Aref K, Reinersman GT, Koenigsberg M, Kogan M, Bello $J$, et al. Diffuse basal ganglia or thalamus hyperechogenicity in preterm infants. $J$ Perinatol 2006;26:230-6.

25. Althabe F, Belizán JM, McClure EM, Hemingway-Foday J, Berrueta M, Mazzoni A, Ciganda A, et al. A population-based, multifaceted strategy to implement antenatal corticosteroid treatment versus standard care for the reduction of neonatal mortality due to preterm birth in low-income and middle-income countries: the ACT 
cluster-randomised trial. Lancet 2015;385:629-39.

26. Roberts D, Dalziel S. Antenatal corticosteroids for accelerating fetal lung maturation for women at risk of preterm birth. Cochrane Database Syst Rev 2006 Jul 19;(3):CD004454.

27. Mwansa-Kambafwile J, Cousens S, Hansen T, Lawn JE. Antenatal steroids in preterm labour for the prevention of neonatal deaths due to complications of preterm birth. Int J Epidemiol 2010;39 Suppl 1:i122-33.

28. Rüegger C, Hegglin M, Adams M, Bucher HU; Swiss Neonatal Network. Population based trends in mortality, morbidity and treatment for very preterm- and very low birth weight infants over 12 years. BMC Pediatr 2012 Feb 22;12:17. doi: 10.1186/1471-2431-12-17.

29. Ballot DE, Potterton J, Chirwa T, Hilburn N, Cooper PA. Developmental outcome of very low birth weight infants in a developing country. BMC Pediatr 2012;12:11.

\begin{tabular}{|c|c|c|c|}
\hline \multicolumn{4}{|c|}{ Table 1. Demographic and clinical details } \\
\hline \multirow[b]{2}{*}{ Demographic/clinical details } & \multicolumn{3}{|c|}{ Study infants $(n=100)$} \\
\hline & & $\begin{array}{l}\%<10 \text { th } \\
\text { centile* }^{*}\end{array}$ & $\begin{array}{l}\text { \%>90th } \\
\text { centile* }\end{array}$ \\
\hline GA, weeks (mean \pm SD) & $28.8 \pm 2.2$ & & \\
\hline GA, weeks (median; range) & $30.25 ; 23.4-34.4$ & & \\
\hline BW, $g($ mean \pm SD) & $1249 \pm 325$ & 21 & 2 \\
\hline Weight at discharge of surviving infants, $\mathrm{g}$ (mean $\pm \mathrm{SD}$ ) & $1896 \pm 229$ & 77 & 0 \\
\hline Length at birth, $\mathrm{cm}$ (mean $\pm \mathrm{SD}$ ) & $37.0 \pm 5.3$ & 35 & 3 \\
\hline Length at discharge home, $\mathrm{cm}$ (mean $\pm \mathrm{SD})$ & $44.0 \pm 1.6$ & 47 & 0 \\
\hline $\mathrm{HC}$ at birth, $\mathrm{cm}$ (mean $\pm \mathrm{SD})$ & $28.0 \pm 2.3$ & 14 & 5 \\
\hline $\mathrm{HC}$ at discharge, $\mathrm{cm}$ (mean $\pm \mathrm{SD})$ & $32.0 \pm 1.4$ & 17 & 3 \\
\hline Clinical chorioamnionitis ${ }^{\star *}(\%)$ & 6 & & \\
\hline Prolonged rupture of membranes (>18hrs) (\%) & 9 & & \\
\hline Mode of delivery: normal cephalic / breech (\%) & $24 / 6$ & & \\
\hline - elective CS/ emergency CS (\%) & $9 / 61$ & & \\
\hline Apgar score at 1 minute (median; range) & $4 ; 1-7$ & & \\
\hline Apgar score at 5 minutes (median; range) & $5 ; 2-8$ & & \\
\hline Twins / triplets (\%) & $19 / 8$ & & \\
\hline
\end{tabular}

This article is protected by copyright. All rights reserved. 


\begin{tabular}{|c|c|}
\hline Male /female (\%) & $57 / 43$ \\
\hline Antenatal steroids given (\%) & 43 \\
\hline Vitamin $\mathrm{K}$ given (\%) & 39 \\
\hline Resuscitated at birth (bag/mask or Neopuff), (\%) & 25 \\
\hline Intubated at birth, (\%) & 3 \\
\hline Initial diagnosis of perinatal asphyxia ${ }^{\star \star \star}(\%)$ & 15 \\
\hline Respiratory distress / congenital pneumonia (\%) & $83 / 17$ \\
\hline Infants treated only with CPAP (\%) & 77 \\
\hline Days on CPAP (median; range) & $3 ; 1-17$ \\
\hline Infants needing mechanical ventilation (\%) & 22 \\
\hline Days on mechanical ventilation (median; range) & $4.5 ; 1-16$ \\
\hline $\mathrm{O}_{2}$ requirement at 36 weeks (chronic lung disease) (\%) & 5 \\
\hline Suspected sepsis $^{\#}$ & 32 \\
\hline Necrotising enterocolitis $^{\# \#}$ (managed conservatively) & 8 (6 died, 2 survived $)$ \\
\hline Received blood transfusion (\%) & 50 \\
\hline Retinopathy of prematurity (III-IV) needing surgery (\%) & 3 \\
\hline Discharged home from the unit (\%) & 73 \\
\hline Transferred to another hospital / orphanage (\%) & 8 \\
\hline Neonatal deaths (\%) & 19 \\
\hline - Age at death, days (median; range) & $11 ; 3-64$ \\
\hline - GA at birth of infants who died (mean \pm SD) & $27.2 \pm 2.4$ \\
\hline - BW of infants who died (mean \pm SD) & $904 \pm 244$ \\
\hline
\end{tabular}

${ }^{*}$ Fenton / WHO growth charts

** Presence of fever $>38^{\circ} \mathrm{C}$ and two other signs: uterine tenderness, maternal or fetal tachycardia and foul /purulent amniotic fluid,

${ }^{* * \star}$ No early spontaneous respiration, being unresponsive or excessively floppy and/or having seizures. In many instances this diagnosis was incorrect

\# At least three clinical or laboratory signs: respiratory distress, temperature instability, feeding intolerance, haemodynamic instability, positive C-reactive protein (CRP), increased immature/total neutrophil ratio, neutrophils with toxic granulation.

\#\# Diagnosed by Bell criteria

BW, birth weight; C, centile; CPAP, continuous positive airway pressure; CS, Caesarean section; GA, gestational age; HC, head circumference; SD, standard deviation

\begin{tabular}{|l|c|c|c|c|}
\hline \multicolumn{5}{|c|}{ Table 2. Main findings seen on early scans (first 10 days after birth) } \\
\hline $\begin{array}{l}\text { Sonographic } \\
\text { findings }\end{array}$ & $\begin{array}{c}\text { Total number } \\
\text { of infants }\end{array}$ & \multicolumn{3}{|c|}{ Incidence of finding depending on age at } \\
scan
\end{tabular}

This article is protected by copyright. All rights reserved. 


\begin{tabular}{|l|c|c|c|c|}
\hline HPI & 7 & 1 & 2 & 4 \\
\hline PVE & 34 & 21 & 7 & 6 \\
\hline
\end{tabular}

* Coded by the highest grade of haemorrhage (some infants had lower grades of GMH-IVH on the first scan and then progressed to a higher grade - these infants are represented twice in columns 3-5, but only once in column 2. Column 2 shows the total number of infants with each diagnosis according to the highest degree of haemorrhage; columns 3-5 show individual scan findings at each period).

GMH-IVH was graded according to Volpe (11): GMH-IVH grade I (GMH): Bleeding confined to periventricular area (germinal matrix); GMH-IVH grade II (IVH II): Intraventricular bleeding (10-50\% of ventricular area on sagittal view); GMH-IVH grade III (IVH III): Intraventricular bleeding (>50\% of ventricular area or distends ventricle).

VM, ventriculomegaly; GMH, germinal matrix haemorrhage; IVH, intraventricular haemorrhage; HPI, haemorrhagic parenchymal infarction; PVE, periventricular echogenicity; $L$, left; $R$, right.

\begin{tabular}{|l|c|}
\hline \multicolumn{2}{|l|}{ Table 3. Sonographic findings at term-equivalent age (n=68 patients) } \\
\hline & N \\
\hline Ventriculomegaly not associated with IVH & 15 \\
\hline Porencephalic cyst & 2 \\
\hline Cysts in caudothalamic notch (and subependymal cysts) & 20 (1) \\
\hline Mild periventricular echogenicity & 17 \\
\hline Cystic periventricular leukomalacia & 2 \\
\hline Post-haemorrhagic ventriculomegaly & 3 \\
\hline Enlarged ECS / IHF & 11 \\
\hline Thin corpus callosum & 5 \\
\hline Apparent reduced volume white matter (2 also with wide IHF/ECS) & \\
\hline Echogenicity in the basal ganglia and/or thalami & \\
\hline Marked: 11 (5 also had wide IHF/ECS, 1-reduced WM volume, 2-thin CC) & \\
\hline Moderate: 14 (5 also had wide IHF/ECS, 1-thin CC) & \\
\hline Mild: 30 (11 also had wide IHF/ECS, 1-reduced WM volume, 1-thin CC) & \\
\hline Lenticulostriate vasculopathy & \\
\hline Normal scan & \\
\hline & \\
\hline
\end{tabular}

This article is protected by copyright. All rights reserved. 
IVH, intraventricular haemorrhage; ECS, extracerebral space, IHF; interhemispheric fissure; WM, white matter; CC, corpus callosum

\section{Figure legends}

Figure 1

The upper images show a. early mid-coronal and b. parasagittal scans from an infant of 24 weeks gestation with mild ventriculomegaly without intraventricular haemorrhage (IVH). The middle image c. shows bilateral IVH seen on day one from an infant of 29 weeks gestation and image d. shows a left-sided haemorrhagic parenchymal infarction seen in an infant of 31 weeks gestation on day five. The lower images show e. posterior coronal and f. parasagittal scans from an infant of 31 weeks gestation on day seven with mild bilateral periventricular echogenicity.

Figure 2

The upper images show in a. atypical bilateral parenchymal haemorrhage (day three) and in b. haemorrhage in the cerebellar vermis with a dilated 3rd ventricle (day 24) from an infant of 32 weeks gestation. There was more extensive haemorrhage in the basal ganglia and white matter (not shown). Image c. shows late diffuse bilateral echogenicity in the basal ganglia from an infant born at 31 weeks gestation and scanned at 36 weeks post-menstrual age.

The lower images are from an infant of 34 weeks gestation and show in $\mathrm{d}$. echogenicity in both caudo-thalamic notches and in e. strands across the posterior horns of the lateral ventricles with some increased white matter echogenicity around the ventricles on day one. Image f. from the same infant on day 39 shows the evolution of cysts in both caudo-thalamic notches. These findings were a common observation in many infants though the echogenicity did not usually appear till three- 
four weeks after birth and the cysts later, but this infant, who also had ventricular strands, was positive for cytomegalovirus.

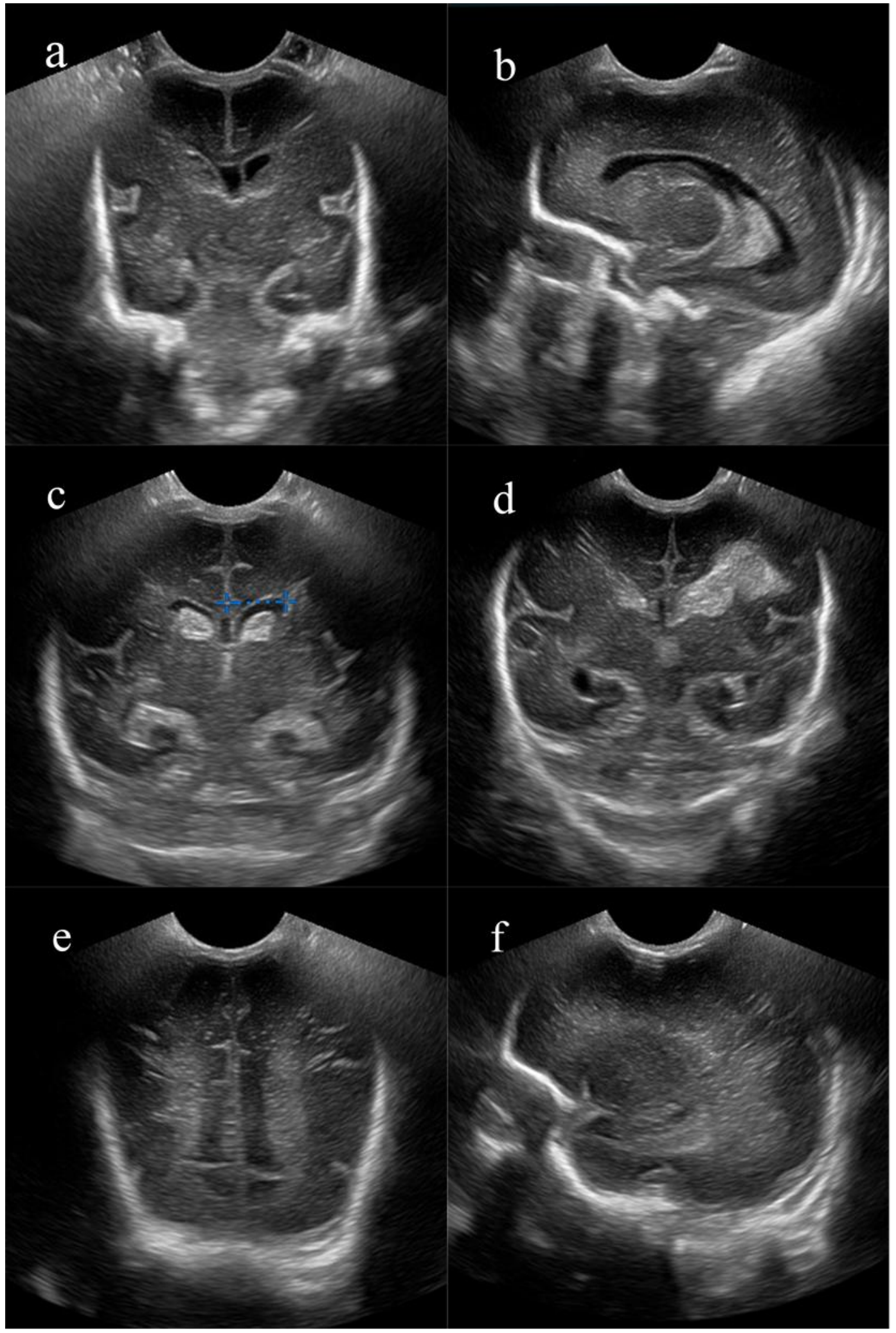

This article is protected by copyright. All rights reserved. 


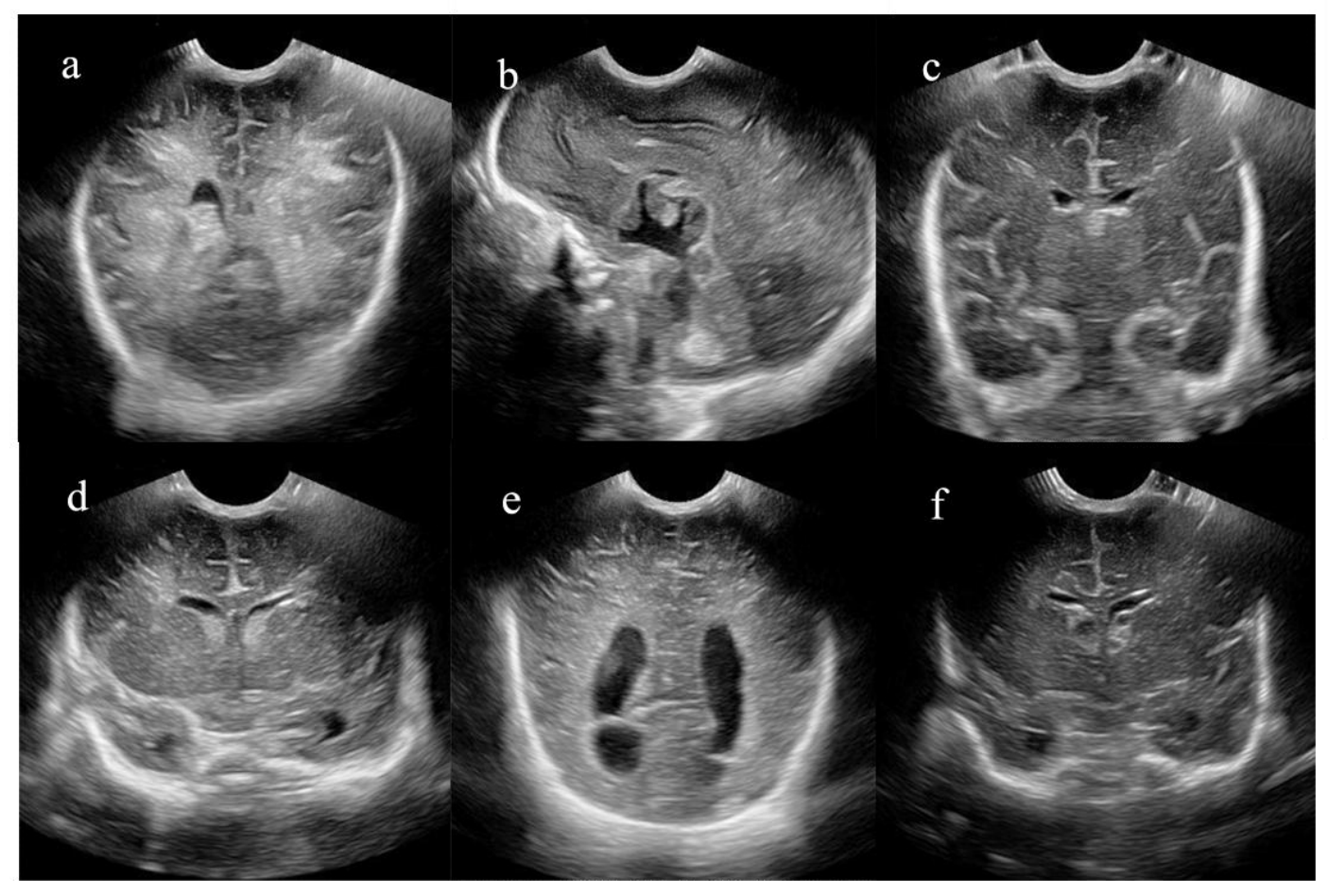

This article is protected by copyright. All rights reserved. 